\title{
Synthesis of Virtual Environments for Aircraft Community Noise Impact Studies
}

\author{
Stephen A. Rizzi ${ }^{*}$ and Brenda M. Sullivan ${ }^{\dagger}$ \\ NASA Langley Research Center, Hampton, VA, 23681-2199
}

\begin{abstract}
A new capability has been developed for the creation of virtual environments for the study of aircraft community noise. It is applicable for use with both recorded and synthesized aircraft noise. When using synthesized noise, a three-stage process is adopted involving non-real-time prediction and synthesis stages followed by a real-time rendering stage. Included in the prediction-based source noise synthesis are temporal variations associated with changes in operational state, and low frequency fluctuations that are present under all operating conditions. Included in the rendering stage are the effects of spreading loss, absolute delay, atmospheric absorption, ground reflections, and binaural filtering. Results of prediction, synthesis and rendering stages are presented.
\end{abstract}

\section{Nomenclature}

$\alpha=$ atmospheric filter absorption coefficient

A $\quad=$ atmospheric absorption

$A_{n} \quad=$ tonal amplitude

$\beta=$ elevation angle

$B P F \quad=$ blade passage frequency

$c \quad=$ speed of sound

$\delta \quad=$ source directivity angle

$d_{a} \quad=$ atmospheric filter absorption distance

$D S B P F=$ Doppler shifted blade passage frequency

$\gamma \quad=$ specific weight

$\lambda=$ acoustic wavelength

$K=$ lapse rate

$L=$ maximum acoustic source dimension

$M \quad=$ Mach number

$\omega=$ frequency

$\Phi_{n} \quad=$ tonal phase

$p \quad=$ pressure

$\vec{P}_{l}(t) \quad=$ observer position

$\vec{P}_{s}(\tau) \quad=$ emission position

$R(\tau) \quad=$ emission distance

$t=$ observer time

$T=$ temperature

$\tau=$ emission time

$z \quad=$ altitude

\footnotetext{
${ }^{*}$ Aerospace Engineer, Structural Acoustics Branch, Mail Stop 463, AIAA Associate Fellow.

${ }^{\dagger}$ Aerospace Engineer, Structural Acoustics Branch, Mail Stop 463.
} 


\section{Introduction}

T psychoacoustic studies at NASA Langley Research Center, human subjects listen to aircraft flyover sounds and Irate them according to various scoring methodologies. The subjective acoustic experiments take place in the field or under controlled laboratory conditions. Field tests using real aircraft flying over the subjects are challenging because it is not known precisely what each listener is hearing, the same flyover can never be reproduced exactly, and flight tests are expensive. Therefore, NASA Langley has historically presented flyover recordings or syntheses for evaluation using loudspeakers in laboratory listening situations. The outdoor environment is typically represented using an anechoic room or an acoustically treated auditorium with up to ten speakers in the walls and ceiling ${ }^{1-4}$. Such laboratory situations allow more control of the test conditions than field-testing. Signals can be exactly replayed, the same sound can be presented at different levels, and predicted sounds from aircraft of the future can be synthesized and played to listeners. However, improvements in the spatial feel and time varying characteristics of the acoustic presentations are needed before the actual flyover sounds can be faithfully reproduced in the artificial laboratory setting. One method is the use of binaural recordings made with a dummy mannequin in a field test and played back to subjects over headphones, avoiding the impurities of the room acoustic playback characteristics. While such an approach gives a convincing presence to some degree, the playback is limited to the fixed orientation in which the recording was made. This prevents the subjects from localizing the sound source by both visual and aural means, turning their heads as they would do in the real world.

In an attempt to retain a controllable test environment, yet more closely recreate conditions in the field, a new capability was developed, ${ }^{5}$ which allows subjects to be immersed both visually and aurally in a three-dimensional, exterior virtual environment. Head tracking (real-time measurement of a listener's head orientation), real time graphics rendering and binaural simulation allow the sense of presence ${ }^{6}$ to be maintained as the subject interacts with the test environment. The use of recorded aircraft flyover noise in that work greatly simplified the simulation because the recordings contain the time varying characteristics associated with the source itself and the propagation from the source to the observer. Under ideal recording conditions, such a simulation is likely the best that can be achieved in the laboratory, as it most closely resembles the environment that listeners are exposed to on a daily basis. From a practical standpoint, however, the simulation is limited in value by the existence of extraneous noise in the recordings (natural or artificial), the finite number of fixed recording positions and the cost of conducting the flight tests. Perhaps the greatest limitation is the inability to examine proposed aircraft, engines, flight procedures, and other conditions or configurations for which, obviously, recorded data are not available. Synthesis of aircraft flyover noise as an alternative to recordings is therefore desirable as it allows the flexibility and freedom to study sounds from aircraft not yet flown.

A sample-based synthesis approach would require static ground measurements or near-field flight measurements of each component under a range of directivity angles and operating conditions. A long time history could be stitched together by cross-fading a number of short time samples. This is however not judged to be practical and any attempt to synthesize a new noise component would be ad hoc. A synthesis approach based on aircraft component noise predictions thus offers advantages over the sample-based approach, assuming suitable prediction tools are available. An earlier aircraft noise synthesizer, ${ }^{7}$ developed for simplified flight paths and component noise sources, was not suitable for synthesizing the sounds required in future studies of low noise flight operations. Instead, a new approach was developed for synthesizing flyover noise, which is suitable for arbitrarily complex sources and flight paths.

The focus of this paper is on the creation of virtual environments using prediction-based synthesized aircraft flyover sounds. It consists of three stages: prediction, synthesis, and rendering. Discussion of the prediction stage is limited to the prediction tool used, not the underlying component noise prediction analyses. An in-depth treatment of the synthesis and rendering stages is presented. In the synthesis stage, component noise source predictions in the form of $1 / 3$-octave band directivity patterns are used to construct pressure time histories at the flying source. Methods for synthesizing broadband and tonal sources are discussed. Also discussed are methods for introducing temporal variations associated with changes in operational state, and low frequency fluctuations that are present under all operating conditions. Included in the rendering stage are the effects of spreading loss, absolute delay, atmospheric absorption, ground reflections, and binaural filtering. The rendering stage can accommodate either recorded or synthesized flyover sounds. Whereas the prediction and synthesis stages are computed a priori, the rendering stage is performed in real-time, allowing creation of an immersive test environment. Results of both 
synthesis and rendering stages are presented. These are available for download via the Internet at http://stabserv.larc.nasa.gov/flyover/. The audio clips are best when heard over headphones.

\section{Virtual Environment Architecture}

A system for creating a virtual environment for simulation of aircraft flyover noise was developed using a combination of custom application software and commercial off-the-shelf hardware/software. A version of the system is shown schematically in Figure 1. The visual simulation is performed on a graphics server running a custom OpenGL Performer ${ }^{8}$ application written using the CAVELib immersive API ${ }^{9}$. This graphics application environment was selected because it allows an identical code base to be run across various computer architectures, operating systems, and visual display environments. The graphics server also acts as a client to the audio server. The master clock for the simulation resides on the graphics server and is used to interpolate the aircraft trajectory for visual rendering, and to provide synchronization with the audio server. For simulation of aircraft flying over ground-based observers, a head mounted display eliminates the need for a six-sided display. Listener orientation tracking is performed by the trackd ${ }^{10}$ daemon application on the graphics server. This information is used by the graphics server for control of the proper visual perspective and by the audio server for proper aural perspective.

The function of the audio server is to represent the time-varying behavior of the acoustic path in a real-time fashion to permit creation of an immersive three-dimensional audio environment. The acoustic path is unique for each source-observer pair. The AuSIM GoldServer ${ }^{11}$ audio server was selected for this application as it allows control of the dynamic path behavior via various empirical or physical models. To accomplish this, time-varying gains, time delays, and filters are applied to the input audio stream, and a binaural output is presented over headphones to the listener, as shown in Figure 2. For the simulations presented in this paper, the input audio stream is delivered as pre-recorded or synthesized waveforms resident on the audio server. For a simulation with real-time pilot input, the synthesis engine would be required to generate a live input audio stream. The gain, time delay and filters are dynamically computed using path-dependent models. The applicable models will be subsequently discussed. Attributes of the models are controlled through the client. The manner in which the models are controlled is dependent on where the input signal is defined, i.e. at the listener for recorded flyovers, or at the source for synthesized flyovers.

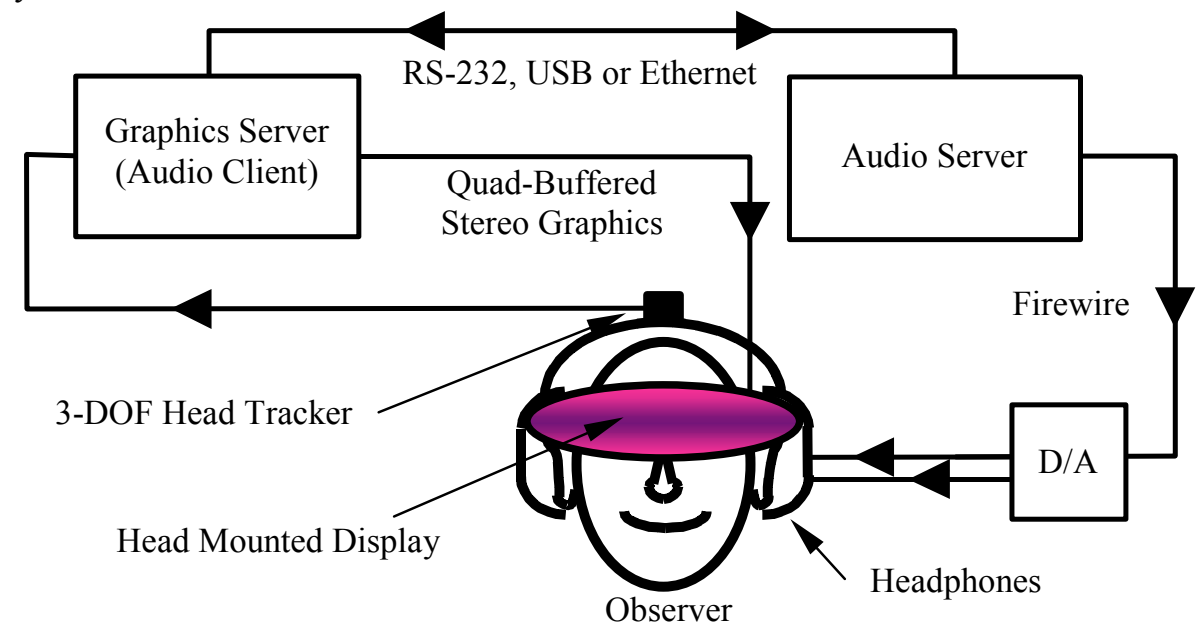

Figure 1: Schematic of a virtual reality architecture.

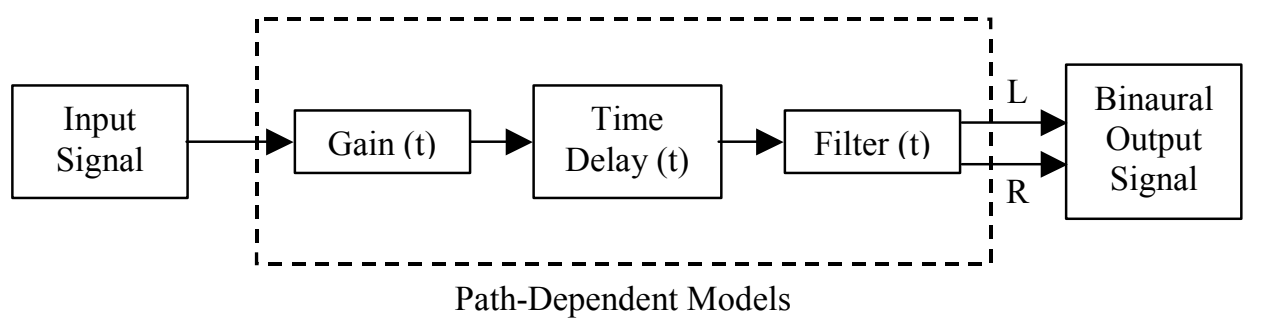

Figure 2: Digital signal processing diagram for audio rendering. 


\section{Audio Rendering Models}

The audio rendering system consists of path-dependent source, environment, material and listener models (see below) each of which contribute gain, time delay, and spectral filters for the aircraft flyover simulation. These quantities are returned to the server digital-signal-processing (DSP) engine, which concatenates and applies them in blocks at a standard audio rate, e.g. $44.1 \mathrm{kHz}$. For multiple sources and a single listener, the binaural output of each path is combined in a mixing stage (not shown) to form a single binaural output for the listener. The audio server runs in a native (non-hardware accelerated) mode. This facilitates development of custom plug-in models, through its open kernel architecture, which can augment or replace the gain, time delay and filter of each model.

In the following, the source is assumed to be compact and is treated as a point source. For the moving source, the maximum acoustic source dimension $L$ must meet the following criteria ${ }^{12}$ :

$$
\begin{aligned}
& L \ll R_{\min } \\
& L \ll \frac{\lambda}{\left|1-M_{r}\right|}
\end{aligned}
$$

where $R_{\min }$ is the minimum distance between the source and observer, $\lambda$ is the shortest wavelength of interest, and $M_{r}$ is the Mach number in the radiation direction.

\section{A. Source-Observer Path}

The path utilized in all path-dependent models is based on the emission position. For a sound observed at time $t$, the emission position is the location of the source at the emission time $\tau$. The emission time is also referred to as the retarded time and is given by

$$
\tau=t-R(\tau) / c
$$

where $c$ is the speed of sound and $R(\tau)$ is the emission distance, i.e. the distance between the source and observer at the emission time. The use of a constant speed of sound dictates a straight-line propagation path, limiting accurate rendering to conditions under which refraction may be ignored. It is convenient to recast equation (2) in terms of a time delay, i.e.,

$$
\operatorname{Delay}(t)=t-\tau=R(\tau) / c
$$

The emission distance may be written in terms of the emission position, $\vec{P}_{s}(\tau)$, and the present observer position, $\vec{P}_{l}(t)$, as

or in terms of the time delay as

$$
R(\tau)=\left|\vec{P}_{s}(\tau)-\vec{P}_{l}(t)\right|
$$

$$
R(t-\operatorname{Delay}(t))=\left|\vec{P}_{s}(t-\operatorname{Delay}(t))-\vec{P}_{l}(t)\right|
$$

Substituting equation (5) in equation (3) gives

$$
\operatorname{Delay}(t)=\left|\vec{P}_{s}(t-\operatorname{Delay}(t))-\vec{P}_{l}(t)\right| / c
$$

Equation (6) must be solved recursively for the time delay, from which the emission position $\vec{P}_{s}(\tau)$ may be found. It should be apparent that equation (6) requires a record of past source trajectory data with a length proportional to the longest delay time. Because the delay almost always occurs at a fraction of the sampling rate, a fractional delay line with sample interpolation is required within the server DSP engine. Two forms of sample interpolation are available on the audio server; linear and bandlimited ${ }^{13}$. The linear interpolation method is computationally fast, but can produce aliasing. The audible effect of aliasing may be somewhat mitigated by the atmospheric absorption filter in the environment model, which reduces the high frequency components. A dynamic polyphase form of bandlimited interpolation eliminates aliasing, but comes at a higher computational expense.

It is also worthwhile to note that only the locations of the source and observer are required in equation (6), not their orientations, i.e. $\vec{P}_{s}(t)=P_{s}(x, y, z, t)$ and $\vec{P}_{l}=P_{l}(x, y, z, t)$. For audio rendering, the orientation of the source is needed only to compute the directivity-dependent gain in the source model, and the observer orientation is required to determine filters used in the listener model. Both models will subsequently be discussed. For the fixed observer simulations undertaken, the observer orientations are measured via a three degree-of-freedom (yaw, pitch, roll) head tracking system. The update rate of the head tracking system is roughly $100 \mathrm{~Hz}$. Source and listener positions and 
orientations are, of course, required by the graphics server to generate the scene graph. However, all visual rendering is based on the source visual position, i.e. the position of the source at time $t$. An example of the visual and emission trajectories for the altitude component of a sample flyover is shown in Figure 3. The delay time is seen to be smallest at the closest position (at about 180s).

\section{B. Standard Source Model}

The standard source model returns the direction-dependent gain for each source. Rotation of the source relative to the listener will cause a change in gain proportional to the source directivity.

\section{Standard Environment Model}

The standard environment model returns the time delay, gain and filter corresponding to the absolute delay, spreading loss and atmospheric absorption, respectively.

\section{Absolute Delay}

Recalculation of the delay in the environment model allows it to be altered, e.g. set to zero, before returning it to the server DSP engine. For absolute delay, the emission distance is computed from the path using equation (4) and the delay from equation (3). Doppler shift is accomplished directly through application of the time varying delay within the server DSP engine. The simulated Doppler shift is proportional to the rate of change of the delay, i.e.,

$$
\frac{\omega}{\omega_{o}}=1-\frac{d}{d t} \operatorname{Delay}(t)
$$

When the delay decreases with time, a positive pitch shift is obtained. When the delay increases with time, a negative pitch shift is obtained. The classic Doppler shift is given by ${ }^{14}$

$$
\frac{\omega}{\omega_{o}}=\frac{1}{1-M_{r}}
$$

where $M_{r}$ is the Mach number in radiation direction at the emission time and position. A comparison of the simulated and classic Doppler shift is shown in Figure 4. Note the asymmetry of the incoming (0-2s) and outgoing (2-4s) shift. The simulated shift would appear symmetric if the delay was incorrectly computed using the observer time rather than the emission time.

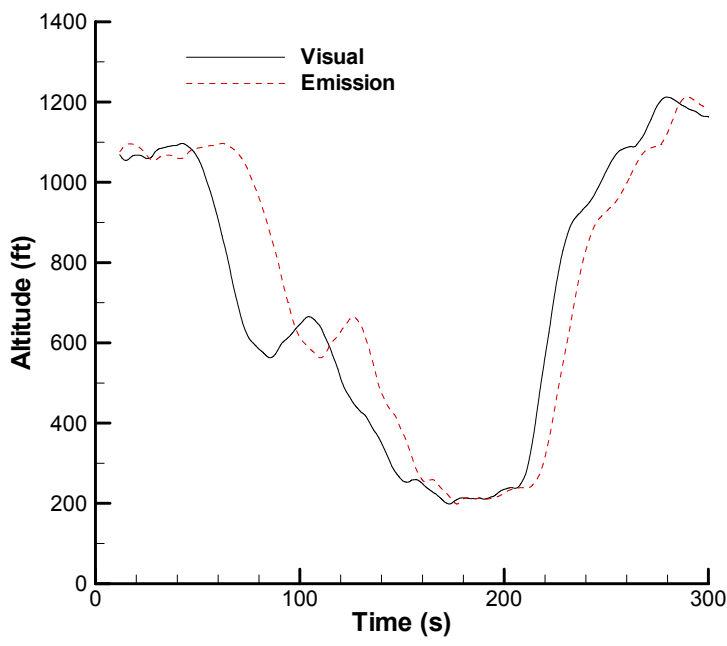

Figure 3: Vertical component of emission and visual flight trajectories.

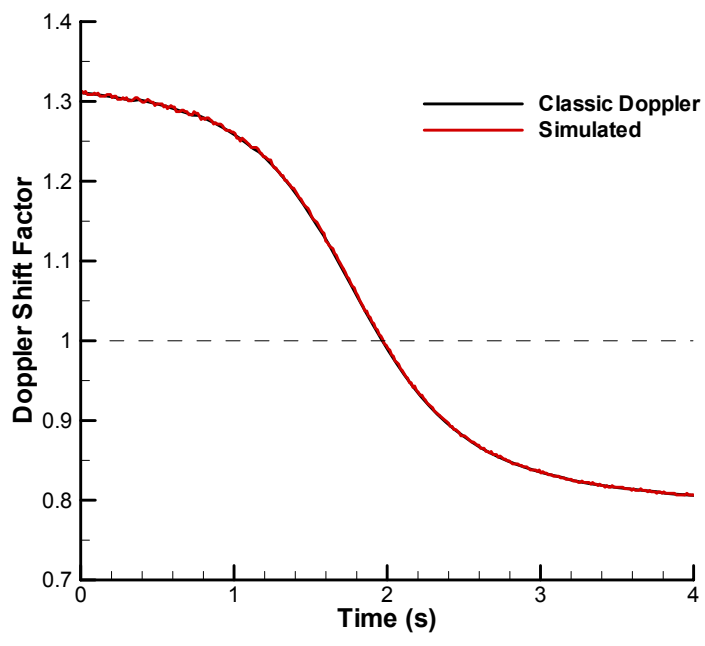

Figure 4: Comparison of simulated and classic Doppler shift for an overhead flyover.

\section{Spreading Loss}

The spherical spreading loss is performed through application of a time varying gain. The gain is computed as

$$
\text { Gain }(t)=\frac{\text { Reference Distance }}{R(\tau)}
$$

The reference distance is the distance from the source at which its amplitude has been defined. 


\section{Atmospheric Absorption}

In the standard environment model, the atmospheric absorption is approximated using a time-varying doubled single-pole infinite impulse response (IIR) filter with a transfer function given by

$$
H(z)=\left(\frac{1-\alpha}{1-\alpha z^{-1}}\right)^{2}, \quad 0 \leq \alpha \leq 1
$$

where the coefficient $\alpha(t)$ is a function of the emission distance $R(\tau)$

$$
\alpha(t)=\frac{R(\tau)}{R(\tau)+d_{a}}
$$

The absorption distance $d_{a}$ is chosen to minimize the weighted error at some target distance. A comparison of the ANSI standard atmospheric absorption ${ }^{15}$ and that obtained by the IIR filter is shown in Figure 5 for a target distance of $121.9 \mathrm{~m}$. The IIR filter generally under-predicts the attenuation at low frequencies $(<2 \mathrm{kHz})$ and over-predicts the attenuation at high frequencies. The attractiveness of this model is not in its accuracy, but in its light computational burden. In addition, since the filter coefficients are determined parametrically, there is no need to interpolate between IIR filters, eliminating the potential of generating an unstable filter.

\section{Improved Environment Model}

An improved environment model was written by the authors to replace the atmospheric absorption filter of the standard environment model with a more accurate representation. The improved model retains the spreading loss (equation (9)) and absolute delay (equation (3)) of the standard model. A secondary purpose was to lay the groundwork for absorption filters that could be used for future simulations with a curved propagation path.

\section{Uniform Atmosphere Absorption Model}

The absorption model for a uniform atmosphere is dependent only on the constant atmospheric conditions (pressure, temperature and relative humidity), the frequency at which it is evaluated, and the straight-line path length $R$ between source and observer. For a selected atmospheric condition, the atmospheric absorption in $\mathrm{dB} / \mathrm{meter}$ is calculated at the 1/3-octave band center frequencies using algorithms by Garber and Willshire ${ }^{16}$, which incorporate the ANSI standard atmospheric absorption ${ }^{15}$, together with methods for calculating saturation vapor pressure ratio proposed by Goff-Gratch ${ }^{15}$ (above $-40^{\circ} \mathrm{C}$ ) and Antoine ${ }^{17}$ (below $-40^{\circ} \mathrm{C}$ ). For each frequency, the total absorption along the path is found by multiplying the absorption in $\mathrm{dB} /$ meter by the path length. The total absorption is thus found for an array of $1 / 3$-octave band center frequencies from $16 \mathrm{~Hz}-10 \mathrm{kHz}$. The dependency of the total absorption with distance for the uniform atmosphere on a standard day $\left(\mathrm{T}=15^{\circ} \mathrm{C}, \mathrm{p}=1 \mathrm{~atm}\right.$.) with $70 \%$ relative humidity is shown in Figure 6. It is seen that the absorption is strongly dependent on range and that the variation is greatest at close range. It is thus necessary to have data at closely-spaced range intervals for accurate specification of close-range absorption.

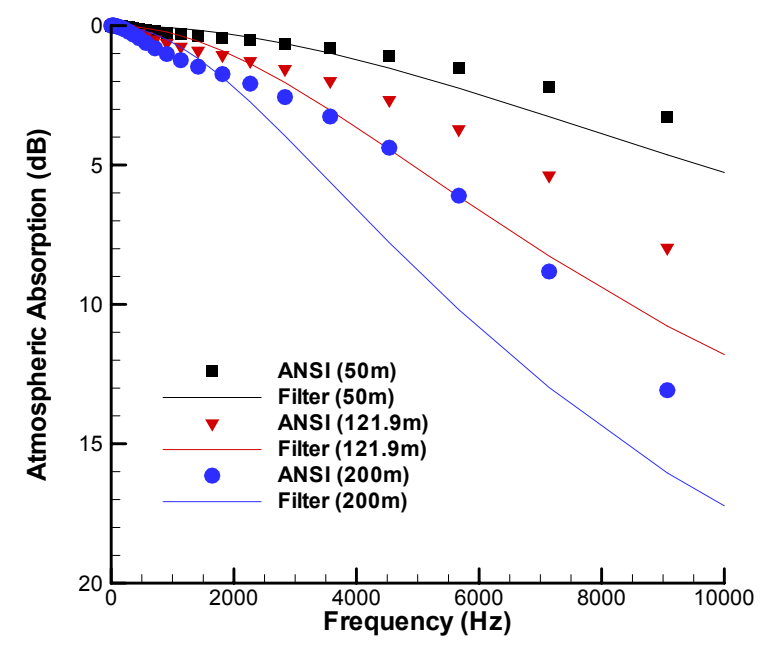

Figure 5: Comparison of ANSI standard atmospheric absorption and approximate IIR filter.

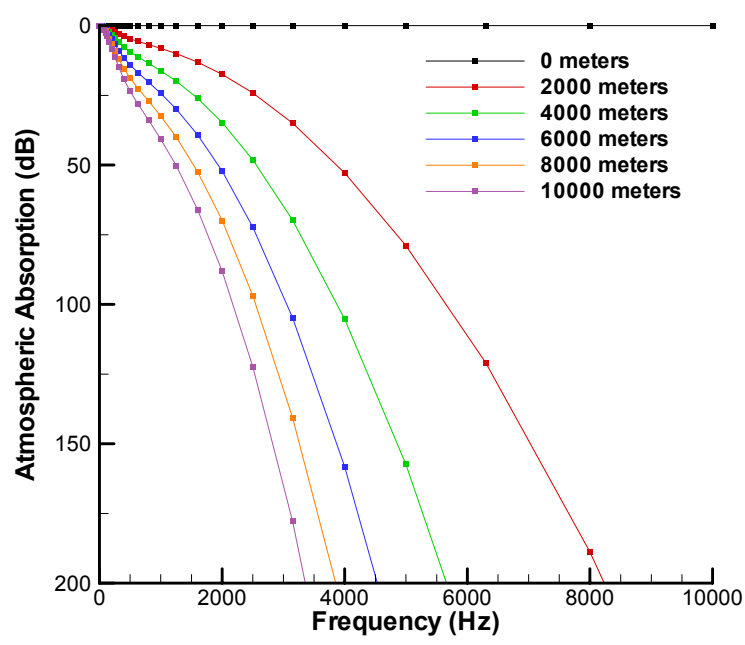

Figure 6: Dependency of atmospheric absorption with distance for a uniform atmosphere. 
A $2^{\mathrm{n}}$ point spline is used to define a curve between the 1/3-octave band center frequencies for each path length specified. This curve is converted to a minimum phase finite impulse response (FIR) filter via an Inverse Fast Fourier Transform (FFT). The absorption at $0 \mathrm{~Hz}$ is defined as zero, as is the absorption at zero distance. By evaluating the total absorption over a range of propagation path lengths, and by determining an FIR filter for each, a one-dimensional atmospheric absorption filter map may be generated.

\section{Non-Uniform Atmosphere Absorption Model}

A two-dimensional filter map may be generated to account for the variation of absorption with altitude in a nonuniform atmosphere. Although refraction will cause the sound to travel in a curved path for this case, a straight-line path is used to integrate the absorption in an approximate manner. The atmospheric absorption between an observer at ground level and a source at some known distance $R$ and angle of elevation $\beta$ with respect to the observer is calculated by first dividing the path into "steps" of equal length, as shown in Figure 7. At the midpoint of each step, the altitude $z$ is found and the atmospheric conditions (pressure, temperature and relative humidity) are evaluated using a particular atmospheric model. For an isothermal atmosphere, temperature $T$ is constant and pressure $p$ varies with altitude according to

$$
p(z)=p_{1} \exp \left[-\frac{\gamma_{1}}{p_{1}}\left(z-z_{1}\right)\right]
$$

where $\gamma$ is the specific weight. For a linear temperature variation with altitude, temperature and pressure vary according to

$$
\begin{aligned}
& T(z)=T_{1}+K z \\
& p(z)=p_{1}\left(\frac{T_{1}}{T_{1}+K z}\right)^{g / K R}
\end{aligned}
$$

where $K$ is the fixed lapse rate, $g$ is the acceleration due to gravity, and $R$ is the gas constant. A constant humidity is usually assumed. Given the pressure and temperature reference values and altitude datum, the atmospheric pressure and temperature at the step midpoints are calculated using equations (12) or (13). For a standard atmosphere, the temperature decreases linearly with increasing altitude with a lapse rate of $-6.5^{\circ} \mathrm{K} / \mathrm{km}$ to an altitude of about $11 \mathrm{~km}$, is isothermal to about $20 \mathrm{~km}$, and then increases in value.

Once the atmospheric conditions at each step midpoint are defined, the atmospheric absorption in $\mathrm{dB} /$ meter at these conditions is calculated at the $1 / 3$-octave band center frequencies using the same algorithms used for the onedimensional case. For each frequency, the total absorption along the path is found by summing the values of absorption in $\mathrm{dB} /$ meter found for each step, $\mathrm{A}_{i}$, multiplied by the step size, $r$, (plus any partial step, $p$ ) for the entire path, or

$$
\mathrm{A}=\sum_{i} r_{i} \mathrm{~A}_{i}+p \mathrm{~A}_{p}
$$

The total absorption is converted into a minimum phase FIR filter using the same procedure presented for the one-dimensional case. By evaluating the total absorption over a range of propagation path lengths and elevation angles, and by determining an FIR filter for each, a two-dimensional atmospheric absorption filter map may be generated.

The variation of atmospheric absorption with range and elevation angle (relative to sea level) is shown in Figure 8 and Figure 9, respectively, for a standard atmosphere on a standard day $\left(\mathrm{T}=15^{\circ} \mathrm{C}, \mathrm{p}=1 \mathrm{~atm}\right.$. at sea level) with $70 \%$ relative humidity. These were computed with a range step size of $10 \mathrm{~m}$. Figure 8 shows that, like the uniform atmosphere, the absorption is strongly dependent on range, with the greatest variation at close range. However, the uniform atmosphere, as seen in Figure 6, has less absorption than the standard atmosphere with the same sea level conditions. Figure 9 shows that that the absorption is only slightly sensitive to changes in elevation angle.

\section{Implementation}

A one- or two-dimensional filter map is computed off-line and stored on the audio server. At present, the range and elevation angle intervals are constant. The number of FIR filter taps used will depend on the level of accuracy desired. Figure 10 shows the character of the filter frequency as a function of the FIR filter size. A 128-tap filter for 
example accurately models the absorption to greater than $85 \mathrm{~dB}$ without significant ripple. It is clear that, given a sufficient number of filter taps, the accuracy offered by the FIR filter implementation is significantly greater than can be obtained by the IIR filter implementation of the standard environment model.

Using the audio server open kernel architecture, a plug-in model in the form of a dynamic link library (DLL) was written and is loaded by instruction from the audio client. Based upon the current path length (range) and elevation angle (if 2-D), the DLL interpolates the filter map to determine the appropriate FIR filter coefficients. These are used in place of the IIR absorption filter of the standard environment model. The fact than an FIR filter is used allows interpolation of the filter map without the possibility of an unstable filter.

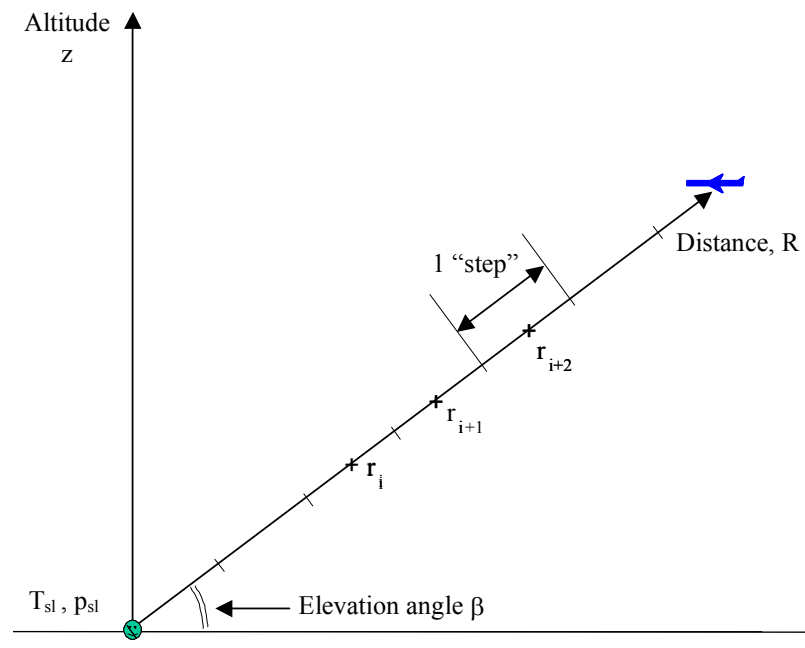

Figure 7: Integration of atmospheric absorption along a straight-line propagation path.

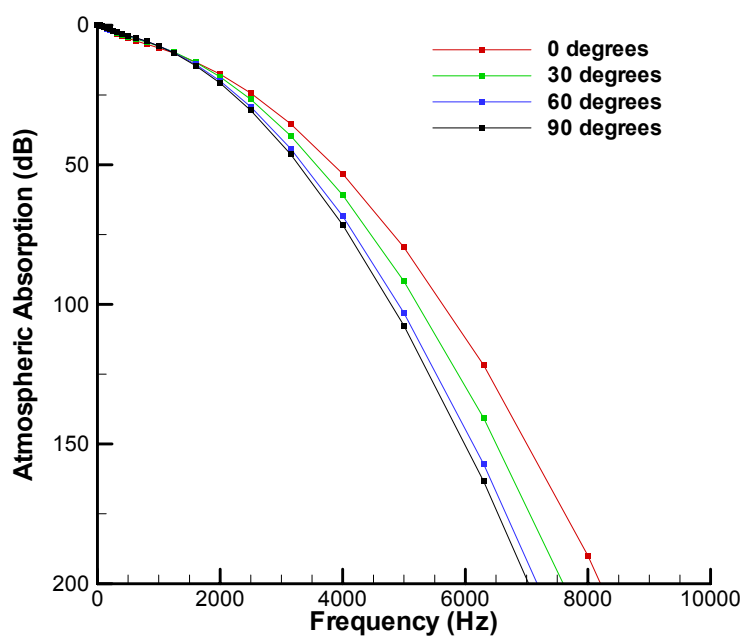

Figure 9: Atmospheric absorption variation with elevation angle at $2 \mathrm{~km}$ for a standard atmosphere.

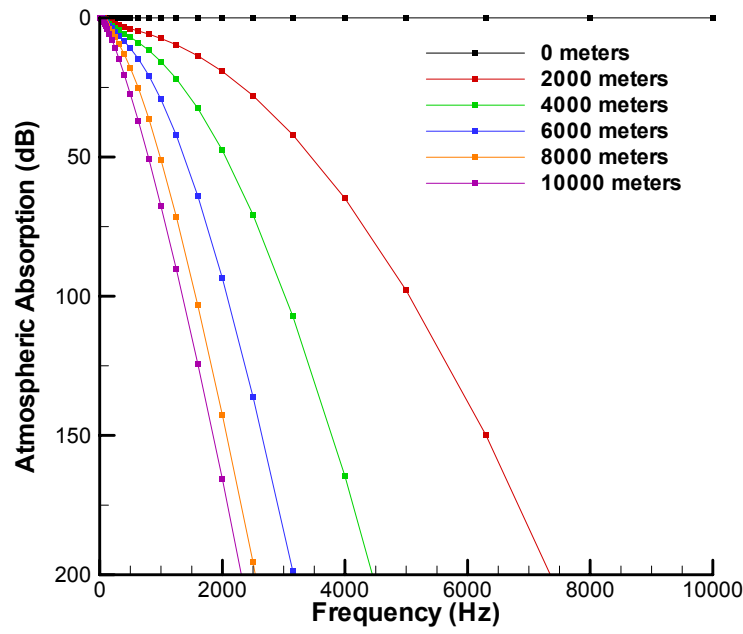

Figure 8: Atmospheric absorption variation with range at a $45^{\circ}$ elevation angle for a standard atmosphere.

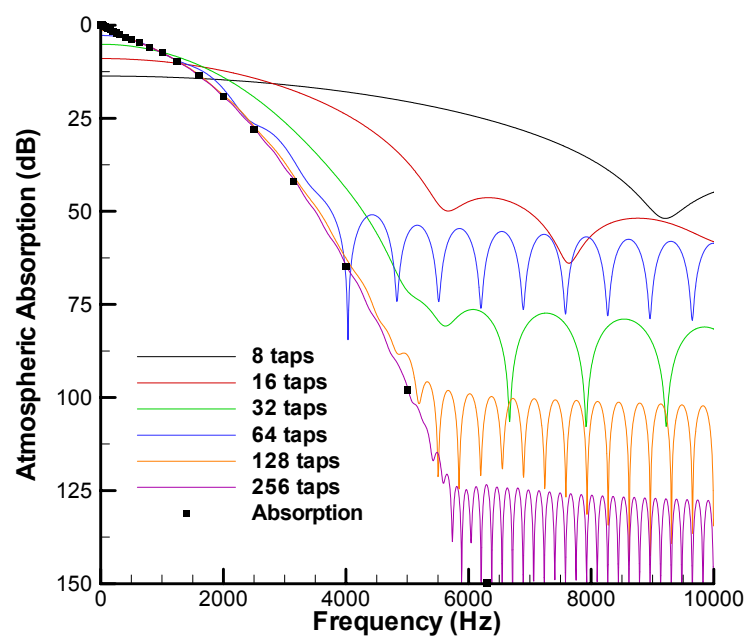

Figure 10: FIR filter frequency response at $2 \mathrm{~km}$ range and $45^{\circ}$ elevation angle for a standard atmosphere.

\section{E. Standard Material Model}

The material model returns a gain term that provides a path independent attenuation through the ground plane. It is used only for virtual (image) sources when modeling ground reflections. A gain of $0 \mathrm{~dB}$ is used to specify a hard surface. The use of the material model is further discussed in section V. 


\section{F. Standard Listener Model}

The listener model returns time delay, gain and filter required to position the source in the virtual threedimensional space about the observer. This process is known as binaural simulation ${ }^{18}$. Head related impulse response (HRIR) functions are applied to the input signal independently for the left and right ears, producing a binaural output stream for presentation over headphones. The HRIR functions account for interaural time delay (ITD), interaural intensity difference (IID), and scattering about the pinnae, head and torso. The latter is represented with minimum phase FIR filters, typically on the order of 128 or 256 taps in length. The particular HRIR used is dependent on the spatial relationship between the source and the observer, specifically the elevation and azimuth angles. This relationship varies with time according to the emission position trajectory, and listener position and orientation. Because the HRIR functions are defined at discrete elevation and azimuth angles, they are interpolated for intermediate angles. This is possible due to their FIR representation.

\section{Simulation of a Recorded Aircraft Flyover}

The creation of virtual environments from recordings provides a mechanism for comparing the quality of synthesized flyovers to recorded flyovers within the same virtual environment. Simulation of a recorded aircraft flyover consists of two stages: recording and rendering. In the following, it is assumed that the aircraft trajectory is recorded via the flight data system or other suitable means, and that a synchronized monaural sound recording is made at a fixed observer location near the ground. The use of recordings greatly simplifies the audio rendering stage because many of the path dependent characteristics are automatically realized in the recordings. Treatment of the signal vis-à-vis the audio server path models is next discussed.

For recorded flyovers, the source directivity pattern is implicit in the recording. The recording may thus be treated within the source model as a monopole having uniform directivity. Therefore, aircraft orientation (yaw, pitch and roll) is required only for visualization.

The gain, time delay, and filter should also be disabled in the environment model because the effects of spreading loss, absolute delay, and atmospheric absorption are part of the recording. Similarly, the presence of a ground surface is also automatically realized in the recording. Therefore, a virtual source is not generated and the material gain is not applicable. In the listener model, the emission position from the path data is used to position the source in the virtual 3-D space.

A master simulation clock running on the graphics server (audio client) synchronizes the visualization with the audio rendering such that a listener in the virtual environment will see the aircraft at the visual position and hear it coming from the emission position. From a programming perspective, at each update cycle the graphics server interpolates the visual trajectories according to the current simulation time, updates the visual scene graph with the interpolated position, and updates the source location on the audio server with the interpolated position and the listener orientation with the current tracking information. Recall that the emission position is calculated on the audio server. Because the amount of information being passed from the graphics server to audio server can get large, particularly for multiple simultaneous renderings, AuSIM developed a capability to preload certain positioning and playback calls within an event list. The event list is essentially a time-stamped sequence of processing events, which are triggered by an internal clock running on the audio server. It is analogous to the processing of note on/off instructions in a music MIDI sequencer. The audio server clock is synchronized with the graphics server master clock so that the two run in parallel. For simulation of recorded flyovers, the visual trajectory is preloaded in an event list along with one instruction to start wave file playback at the appropriate point in time. Once the clocks are synchronized, only the listener orientation data from the head tracking system is passed to the audio server from the graphics server.

\section{A. Masking}

As indicated in the introduction, recordings are often corrupted with extraneous noises. For example, many field recordings contain nearby traffic noise, domestic animal sounds, and insect noise. Since the purpose of the simulation using recorded flyover sound is to make it appear to come from a flying airplane, any extraneous noises will also appear to fly in the simulation.

While it may be possible to filter out some of the extraneous noise, doing so will likely detract from the flyover sounds as these often have broad frequency content, particularly the jet and airframe noise components. For 
stationary sources, an effective means of diminishing one's ability to localize the extraneous flying noise is to mask it with similar non-flying sources. This can be accomplished by positioning several similar recordings, made without the aircraft, about the listener in the virtual environment. The ability to localize on the masking sounds can be further diminished by positioning them in fixed positions relative to the listener. As the listener orientation changes, the location of the masking sounds change as well.

Simulations of recorded flyovers 5 in the form of AVI movies are available as Web clip 1.

\section{Simulation of a Synthesized Aircraft Flyover}

Simulation of a prediction-based synthesized aircraft flyover consists of the following stages: prediction, synthesis, and rendering. The prediction stage is largely outside the scope of this paper. The synthesis stage is considered in section VI. In the following discussion, it is assumed the flyover sound has been synthesized at the source location along a known trajectory. Therefore the real-time audio rendering stage is more complicated than for recordings because each path dependent characteristic must be simulated. Treatment of the signal through the various audio server path models is next discussed.

For synthesized flyovers, the source directivity pattern must be explicitly taken into account. The paradigm used in this work takes the source directivity into account within the synthesis stage, as described in section VI. Therefore, like the recorded flyover, the input audio stream implicitly contains the effect of source directivity and the source may be treated as a monopole. Other synthesis methods may require the use of the source model directivity explicitly.

Within the environment model, the absolute delay is computed from equations (3) and (4). Because the simulated Doppler shift is proportional to the time rate of change of the delay line (see equation (7)), abrupt changes in the derivative may produce undesired audible artifacts in the form of frequency sweeps. The event list helps in this regard because it removes timing jitter in the source position updates from the client. With event list processing, there is no delay in execution of commands; they are executed precisely at the scheduled moment. The spreading loss is computed from equation (9) using the emission distance. Either the standard or improved atmospheric absorption filter is also computed using the IIR or FIR filters, respectively. When using the improved model, the DLL must be loaded prior to rendering. The listener model selects the appropriate HRIR function based on the path data. A schematic depicting the process for simulating flyover noise is shown in Figure 11. The only difference between simulating a recorded flyover and a synthesized flyover is the differing input audio streams and the complexity of real-time rendering.

\section{A. Ground Reflections}

The presence of a ground surface produces a reflection, which interferes with the direct sound. The interference produces a comb filter effect ${ }^{19}$, which alters the spectral content in a time-varying manner as the aircraft moves along its trajectory. Instead of implementing ground reflection as a comb filter within the environment model, an image source is used to spawn a secondary path with its own emission position. The image source is positioned at a location symmetric about the ground plane, as shown in Figure 12. A schematic of the signal processing path is shown in Figure 13. The source, environment and listener models for the direct path operate on the original aircraft trajectory. The source, environment and listener models for the reflected path operate on the imaged aircraft trajectory. For an observer above the ground plane, the reflected path is longer than the direct path. Hence, the delay obtained from the environment model for the reflected path is greater than that of the direct path. The comb filter effect manifests itself at the mixing stage. In its current implementation, the improved environment model uses the emission position of the direct path. For an observer near the ground, the added absorption over the additional path length is insignificant in the audible frequency range. The material model for the reflected path additionally applies the material gain. Because the listener model for the reflected path utilizes the emission position of the imaged source, the reflected sound appears to come from the ground. The input audio stream is common to both the direct path and reflected path.

Recall that for the stationary source, the delay is constant and equal to $R / c$. Therefore, a comb filter produced for the stationary source will be symmetric about the overhead position. For the moving source however, the delay is not symmetric about the overhead position, as was shown in Figure 4. Therefore, the comb filter produced by the moving source will be asymmetric about the overhead position. Unlike the Doppler shift, which always decreases 
the apparent frequency, the "teeth" of the comb filter come closer together on the in-bound portion, and spread apart on out-bound portion of the flyover. This is apparent in the spectrogram of a white noise source "flown" along a straight and level trajectory at an altitude of $121.9 \mathrm{~m}(400-\mathrm{ft}$.) and speed of $78.2 \mathrm{~m} / \mathrm{s}(152 \mathrm{knots})$ over a $1.52 \mathrm{~m}(5-\mathrm{ft}$.) tall observer, see Figure 14. The ground surface was treated as hard, i.e. with a material gain of $0 \mathrm{~dB}$. The effects of spreading loss and atmospheric absorption were disabled in this rendering. The sound may be heard in Web clip 2.

Recorded Monaural Sound at Listener Position or Synthesized Monaural Sound at Source Position

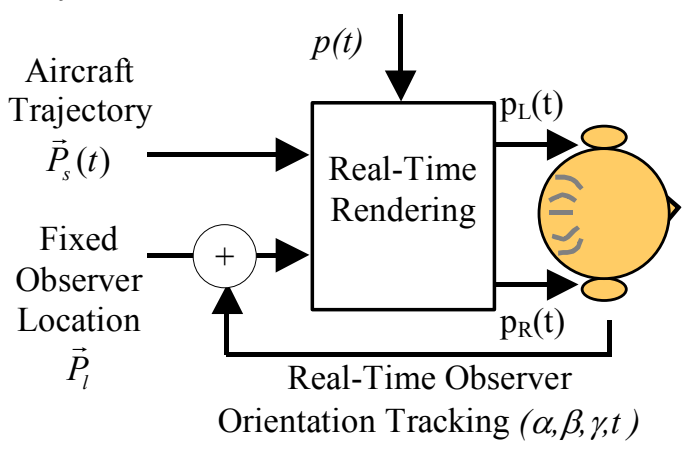

Figure 11: Process for acoustic simulation of flyover noise.

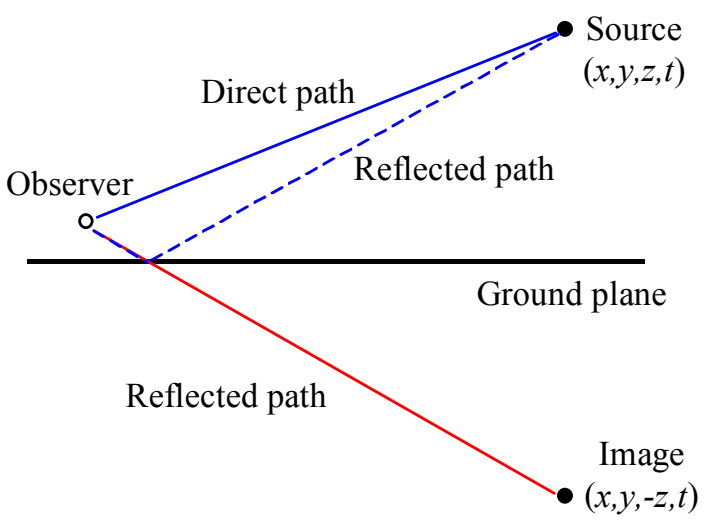

Figure 12: Ground reflection modeling using an image source.

Direct Path Used Source Emission Position

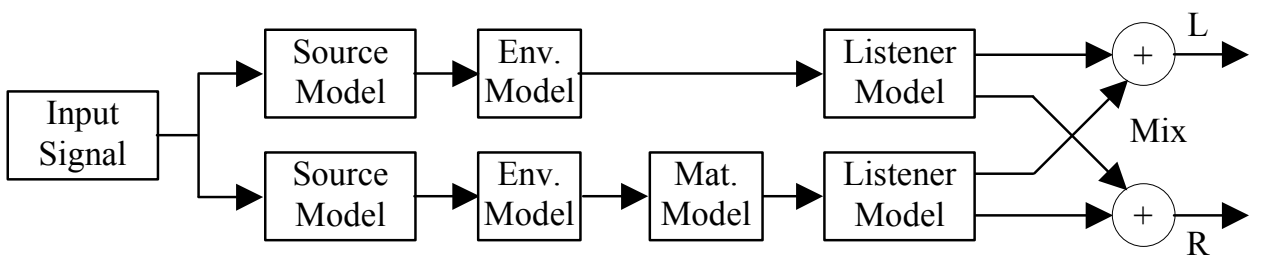

Reflected Path Uses Image Source Emission Position

Figure 13: Signal path for simulation with ground reflection.

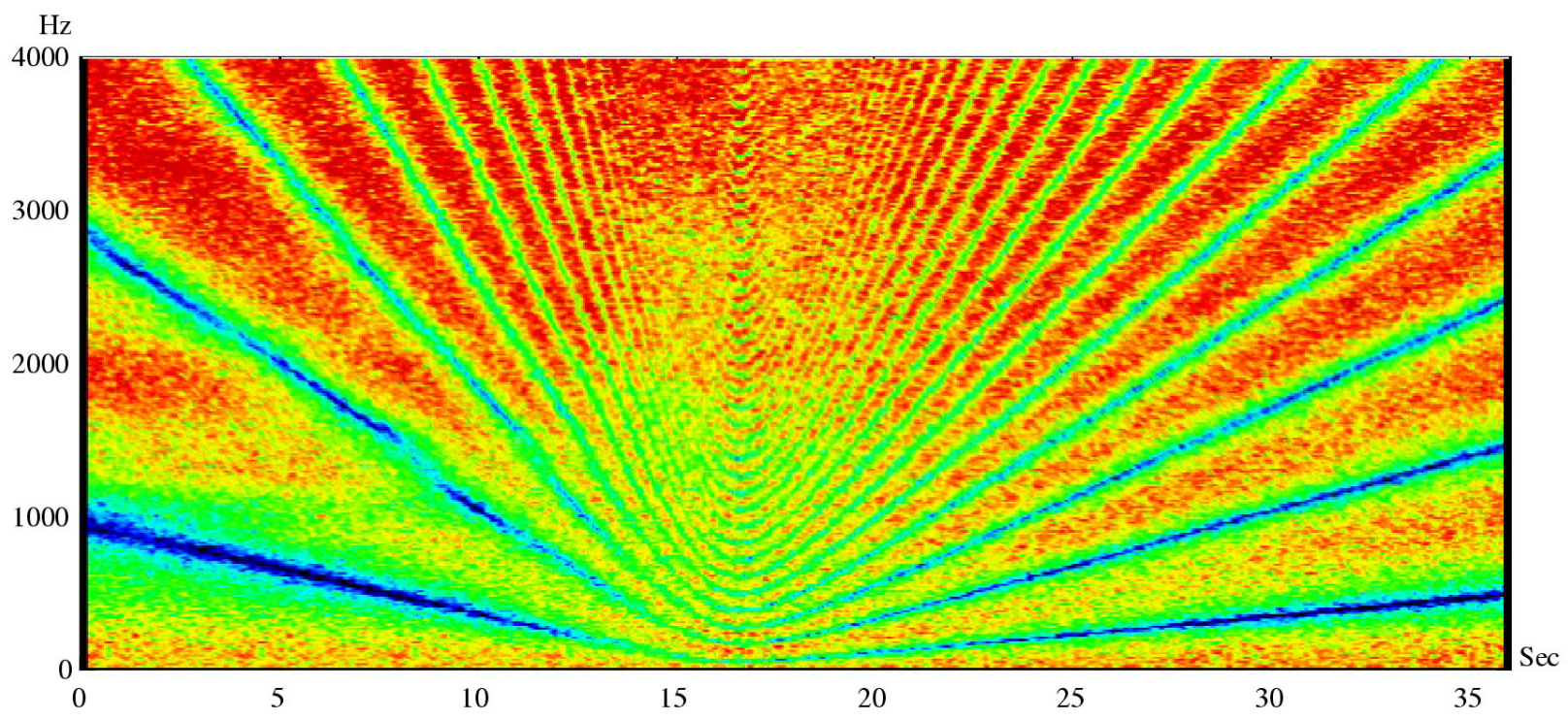

Figure 14: Spectrogram of a simulated overhead flyover with ground reflection. 


\section{Component Noise Synthesis}

Component noise synthesis is performed via one of two approaches. For broadband sources such as jet noise, broadband fan noise and airframe noise, a subtractive synthesis ${ }^{20}$ approach is taken. For tonal sources such as forward/aft radiated fan tones and turbine tones, an additive synthesis ${ }^{20}$ approach is taken. In each case, source directivity predictions obtained from ANOPP $^{21}$ (Aircraft Noise Prediction Program) serve as input to the synthesis. The predictions are made at discrete times along the trajectory when a change in the aircraft operational state occurs. Such a change would include a change in throttle, flaps, etc., but not a change in the aircraft position itself. The directivity pattern used in the synthesis is linearly interpolated in time between each specified operational state to obtain the instantaneous source directivity at the emission time.

Instead of synthesizing the sound at all possible directivity angles, synthesis is performed only at the directivity angle corresponding to a straight-line propagation path between the source and observer at emission time. A diagram of the synthesis strategy is shown in Figure 15. Since the source directivity is predicted at a finite number of discrete angles, a second interpolation of source directivity is performed to obtain the source spectrum at the instantaneous directivity angle. The asterisk symbols in Figure 15 indicate the interpolated directivity angles. The synthesis performed is analogous to what a microphone would record if it were placed near the source along the straight-line propagation path between the observer and the source at the time of emission. As the source moves, the microphone would pick up a new position on the source directivity pattern. In this manner, the source directivity is embedded in the synthesized signal and does not need to be represented within the source model at the time of rendering. This approach to synthesis makes simulation of arbitrary trajectories straightforward.

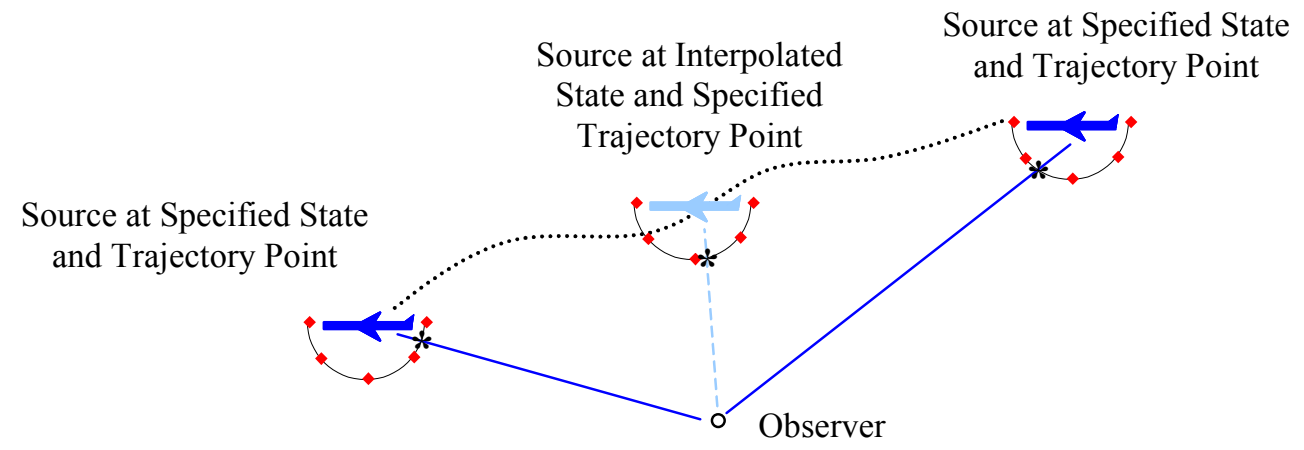

Figure 15: Synthesis is performed between specified aircraft operational states at emission time directivity angle *.

\section{A. Broadband Synthesis}

Source directivity predictions for broadband sources obtained from ANOPP are expressed in 1/3-octave bands as a function of forward-to-aft directivity angle for axisymmetric sources, and in terms of both directivity angle and azimuth angle for non-axisymmetric sources. As an example, Figure 16 shows the axisymmetric source directivity obtained from the Stone jet model ${ }^{22,23}$ in ANOPP, where the directivity angle is measured along the jet axis and 0 degrees is on the forward arc.

\section{Spatial Variations}

The interpolated directivity at emission time is converted to a $2^{\mathrm{n}}$-point narrowband spectrum by dividing the total power within each 1/3-octave band by the number of narrowband bins that fall in each $1 / 3$-octave band. The number of points used determines the lowest frequency that can be represented. An inverse FFT is applied to the narrowband spectrum to obtain FIR filter coefficients. In the present implementation, a pink noise source is convolved point-by-point with the FIR filter in the time domain for a fixed buffer length (typically around 500 points at an audio sampling rate of $44.1 \mathrm{kHz}$ ). By keeping the buffer length small, audible clicks or pops are eliminated because filter changes between buffers are kept small. The speed of this operation could be substantially increased in the future by performing the convolution in the frequency domain in an overlap-add fashion. The spectra of a measured jet noise and a synthesized jet noise are shown in Figure 17 for various size FIR filter lengths ranging from 4096 to 32768 taps. Above 8192 taps, the spectrum of the measured source is accurately recreated. Corresponding audio clips for the measured data and synthesized jet noise with an 8192-tap FIR filter are provided in Web clips 3 and 4, respectively. 


\section{Temporal Variations}

Because the source directivity predictions from ANOPP are computed from time-averaged models, the synthesized source time histories lack some of the temporal variations observed in measured data. Those variations associated with changes in the aircraft operational state occur over long time scales and are represented by changes in the directivity pattern as a function of time. The variations of interest here are those that occur on a shorter time scale at any operating condition. A study was undertaken to characterize the low-frequency $(<20 \mathrm{~Hz})$ fluctuations by performing Short-time FFT analyses using measured jet noise data under steady state operating conditions. ${ }^{24}$ The variation in each $1 / 3$-octave band can be used to amplitude modulate the interpolated source directivity prior to conversion to narrowband. The $1 / 3$-octave band spectrogram of measured jet noise, synthesized jet noise without temporal variation, and synthesized jet noise with temporal variation are shown in Figure 18 - Figure 20. An audio clip for the synthesized jet noise with an 8192-tap FIR filter and low frequency amplitude modulation is provided in Web clip 5.

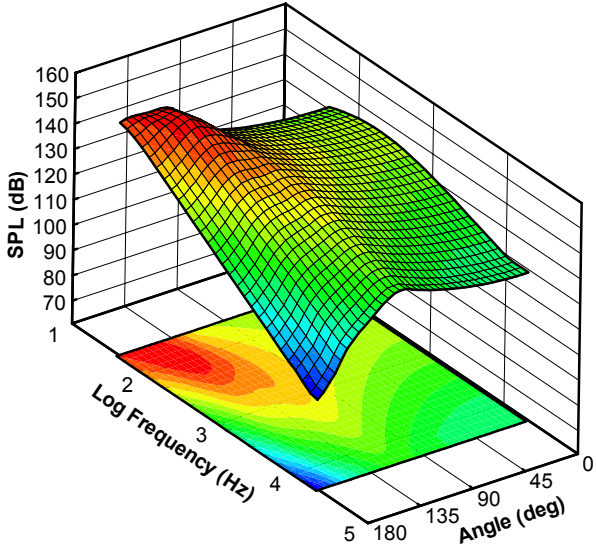

Figure 16: Typical source directivity pattern from ANOPP Stone jet module.

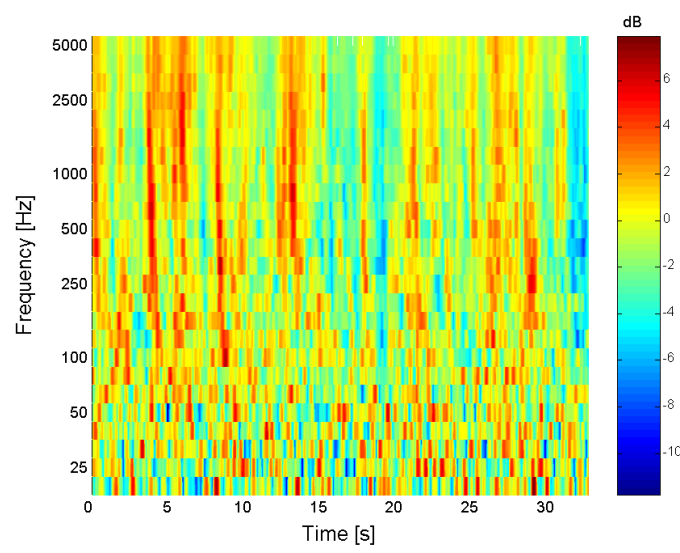

Figure 18: Spectrogram of measured jet noise data.

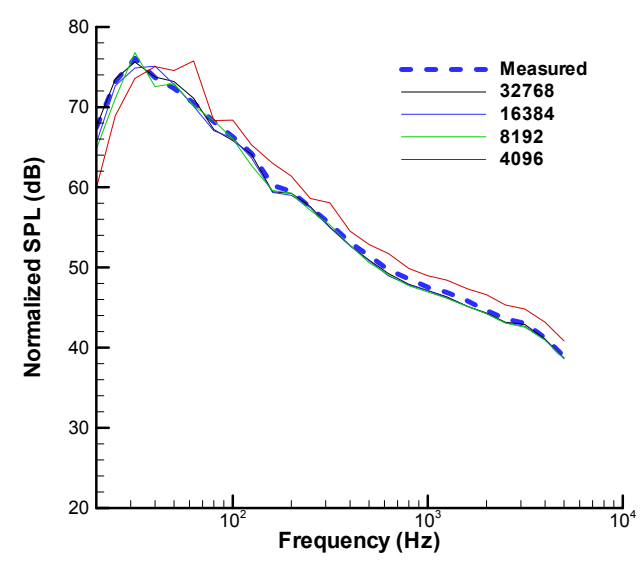

Figure 17: Spectra of measured and synthesized sources.

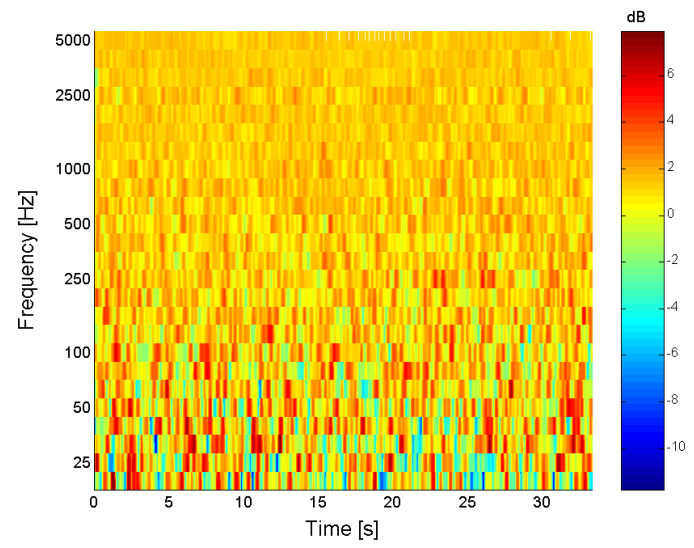

Figure 19: Spectrogram of synthesized jet noise without inclusion of temporal variations.

\section{B. Tonal Synthesis}

Like the broadband directivity, source directivity predictions for tonal sources obtained from ANOPP are expressed in 1/3-octave bands. As an example, Figure 21 shows the source directivity obtained from the Heidmann fan model $^{25}$ in ANOPP. Unfortunately, this form is inconvenient for fan noise as it is both Doppler shifted and potentially contains multiple harmonics in each $1 / 3$-octave band. Therefore, some pre-processing is required. Knowing that the blade passage frequency (BPF) is equal to the number of fan blades times the rotational speed, the Doppler shifted BPF (DSBPF) is:

$$
\mathrm{DSBPF}=\mathrm{BPF} /(1-\mathrm{M} \cos \delta)
$$


where $\mathrm{M}$ is the Mach number and $\delta$ is the specified ANOPP output directivity angle. The DSBPF is computed for each output directivity angle. This frequency falls into one of the 1/3-octave bands of the ANOPP output and the level in that band is assigned to the fundamental tone. For each of the harmonics of the DSBPF, the 1/3-octave band into which it falls is similarly found, and the appropriate band level is assigned to that harmonic, as shown in Figure 22. In cases where multiple harmonics fall into the same $1 / 3$-octave band, the energy in that band is divided between the tones, in a ratio determined by inspection of the complete data set. This computation is repeated for each directivity angle as shown in Figure 23.

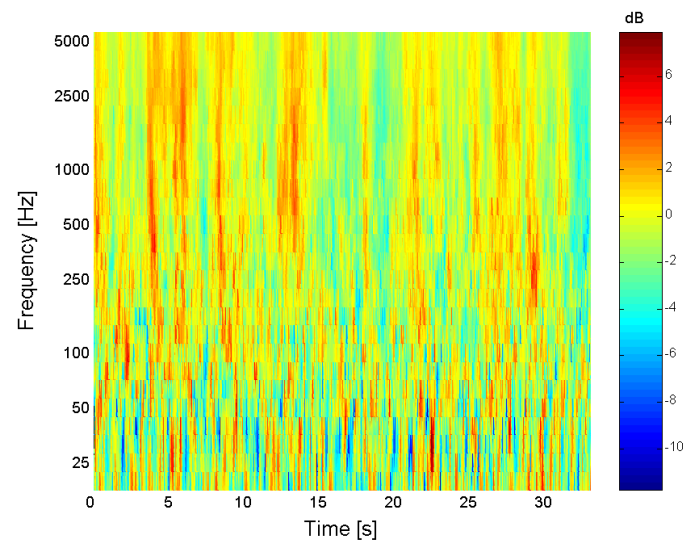

Figure 20: Spectrogram of synthesized jet noise with inclusion of temporal variations.

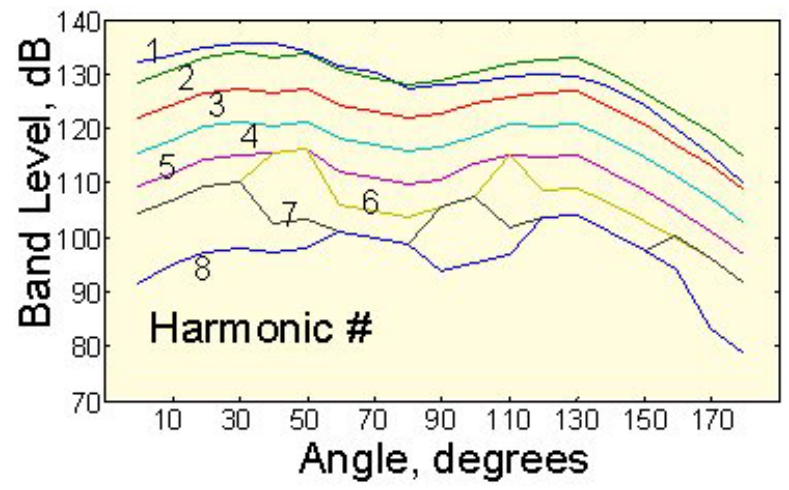

Figure 22: Doppler shifted blade passage frequency harmonics in each $1 / 3$-octave band.

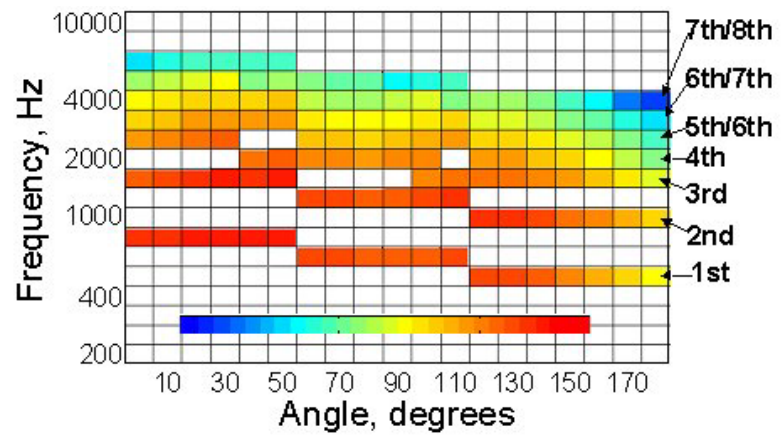

Figure 21: Typical ANOPP HDNFAN module output.

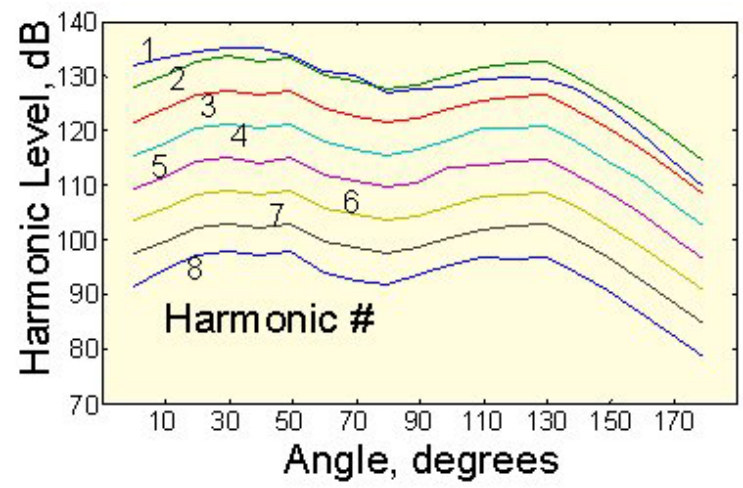

Figure 23: Corrected directivity for blade passage frequency harmonics.

\section{Spatial Variations}

Like the broadband synthesis, the source directivity is linearly interpolated between angles. Unlike the broadband synthesis, the tonal synthesis cannot be performed in a piecewise continuous fashion on a fixed buffer because doing so would introduce audible discontinuities. Instead, the tonal frequencies are tracked between changes in aircraft state and swept in a continuous fashion. Also, tonal amplitudes are linearly varied between the beginning and end of the synthesis buffer. The tonal synthesis is performed by summing sinusoidal components:

$$
p(t)=\sum_{n} A_{n}(t) \sin \left(\omega_{n}(t) t+\Phi_{n}(t)\right)
$$

where the time variation of the tonal amplitude $A_{n}$, frequency $\omega_{n}$, and phase $\Phi_{n}$ may be due to either changes in state, or fluctuations at a particular state. For harmonic sources, equation (16) becomes

$$
p(t)=\sum_{n} A_{n}(t) \sin \left(n \omega_{1}(t) t+\Phi_{n}(t)\right)
$$


where $\mathrm{n}$ is the harmonic number. In the above, the source directivity obtained from ANOPP provides the tonal amplitude and frequency for each aircraft state (as in Figure 23), while empirical data may be used to provide information about fluctuation at a particular state.

\section{Temporal Variations}

A study was undertaken to characterize the low-frequency $(<20 \mathrm{~Hz})$ fluctuations by performing Short-time FFT analyses using measured fan data. ${ }^{24}$ The narrowband variation of amplitude and frequency was characterized. It was determined that the frequency variation fell below the "just noticeable variation in frequency" (jnvf) of a pure tone ${ }^{26}$ and can be ignored in the synthesis. However, amplitude variations were significant and different between harmonics, as shown in Figure 24 and Figure 25. Therefore, the character of the waveform changes over time, and this evolution is an important factor in creating realistic synthesized fan tones. This modulation is not presently implemented in the tonal synthesis.

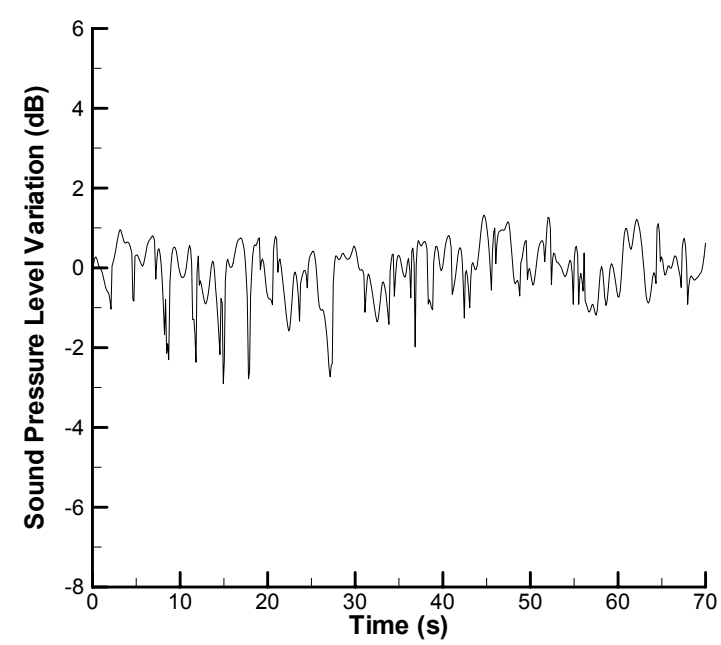

Figure 24: Measured sound pressure level variation about the time average for second harmonic of BPF.

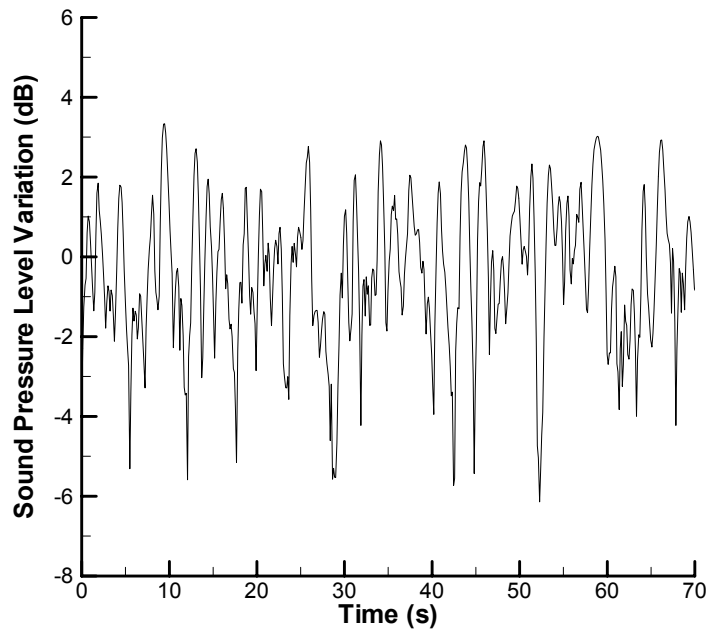

Figure 25: Measured sound pressure level variation about the time average for fifth harmonic of BPF.

\section{Synthesized Aircraft Flyover Renderings}

Simulations of aircraft flyovers using synthesized aircraft component noise were made for a straight and level trajectory at an altitude of $121.9 \mathrm{~m}$ (400-ft.) and constant speed of $78.2 \mathrm{~m} / \mathrm{s}(152 \mathrm{knots})$. A $1.52 \mathrm{~m}(5-\mathrm{ft}$.) tall observer standing on a hard surface was positioned such that the aircraft flew directly overhead from $1.61 \mathrm{~km}$ (1-mi.) to the left to $1.61 \mathrm{~km}$ to the right. The atmospheric absorption filter used was for a standard atmosphere on a standard day with $70 \%$ relative humidity. In the synthesis, two identical aircraft states were specified at the beginning and end of the trajectory. Source directivity predictions were made using ANOPP for jet noise and inlet/aft fan components. The jet noise component was synthesized with low frequency amplitude modulation. The fan noise components were synthesized without low frequency amplitude modulation.

The effects of various rendering models on the simulation were studied by enabling each in a sequential fashion. Each rendering is provided as an audio Web clip. The jet noise component synthesis at the source location is provided in Web clip 6. The effect of spreading loss and binaural simulation on the jet noise component is provided in Web clip 7, the addition of atmospheric absorption is provided in Web clip 8, and the addition of absolute delay is provided in Web clip 9. Note that the effect of Doppler shift is not discernible for this broadband noise source. The additional simulation of ground reflection is provided in Web clip 10. Finally, the addition of the synthesized fan tone source component in Web clip 11 indicates both the Doppler shift resulting from absolute delay and amplitude modulation as the tones pass through the various "teeth" of the comb filter produced by ground reflection.

\section{Concluding Remarks}

A means of creating virtual environments for simulation of aircraft flyover noise has been developed. Because both the synthesis and rendering stages utilize empirical and physical models, the range of applicability is broad and 
is limited only by the sophistication of the models used. Therefore, virtual environments may be produced using the latest component source noise and propagation models available. In this respect, the approach taken is uniquely qualified for studying community noise impact of future flight vehicles and their operations. The near term use will be directed at investigating the perceived benefit of continuous descent approach operations.

Future developments in the near term include implementation of low frequency temporal variations within the tonal synthesis, and calibration of the various source component levels at the time of synthesis. Possible longer-term developments include increasing the efficiency of the synthesis to permit auralization of real-time changes to flight operations, i.e. pilot-in-the-loop scenarios, and an ability to model curved propagation paths to permit more accurate long-range propagation where the effect of temperature gradients and wind cannot be ignored.

\section{Acknowledgements}

The authors wish to acknowledge William L. Chapin, Bryan A. Cook, Kamil Dziekanowski, and Agnieszka Roginska, of AuSIM, Inc. for their excellent support in developments related to the GoldServer audio server.

\section{References}

${ }^{1}$ McCurdy, D.A. and Powell, C.A., "Annoyance caused by propeller airplane flyover noise," NASA TP 2356, August 1984.

${ }^{2}$ McCurdy, D.A., "Annoyance caused by advanced turboprop aircraft flyover noise, single-rotating-propeller configuration," NASA TP 2782, March 1988.

${ }^{3}$ McCurdy, D.A., "Annoyance caused by advanced turboprop aircraft flyover noise, counter-rotating-propeller configuration," NASA TP 3027, September 1990.

${ }^{4}$ Leatherwood, J.D. and Sullivan, B.M., "A laboratory study of subjective annoyance response to sonic booms and aircraft flyovers," NASA TM 109113, May 1994.

${ }^{5}$ Rizzi, S.A., Sullivan, B.M., and Sandridge, C.A., "A three-dimensional virtual simulator for aircraft flyover presentation," ICAD 2003, Proceedings of the 9th International Conference on Auditory Display, Boston, MA, 2003, pp. 87-90.

${ }^{6}$ Witmer, B.G. and Singer, M.J., "Measuring presence in virtual environments: A presence questionnaire," Presence: Teleoperators and Virtual Environments, Vol. 7, No. 3, 1998, pp. 225-240.

${ }^{7}$ McCurdy, D.A. and Grandle, R.E., "Aircraft noise synthesis system," NASA TM-89040, February 1987.

${ }^{8}$ Eckel, G. and Jones, K., "OpenGL Performer Programmer's Guide," Silicon Graphics, Inc., 2000.

9"CAVE Library User's Guide," VRCO, Inc., 2002.

${ }^{10}$ "Trackd User's Guide," VRCO, Inc., 2002.

${ }^{11}$ "GoldServe, AuSIM3D Gold Series Audio Localizing Server System, User's Guide and Reference, Rev. 1d," AuSIM Inc. Mountain View, CA, October 2001.

${ }^{12}$ Farassat, F., "Theory of noise generation from moving bodies with an application to helicopter rotors," NASA TR R 451, December 1975.

${ }^{13}$ Smith III, J.O., "MUS420/EE367A Lecture 4A, Interpolated delay lines, ideal bandlimited interpolation, and fractional delay filter design," Center for Computer Research in Music and Acoustics (CCRMA), Stanford University, Stanford, CA 2005.

${ }^{14}$ Pierce, A.D. Acoustics, An introduction to its physical principles and applications. Acoustical Society of America, Woodbury, NY, 1994.

${ }^{15}$ "American National Standard Method for the calculation of the absorption of sound by the atmosphere," American Institute of Physics, ANSI S1.26-1995 (ASA 113-1995), 1995.

${ }^{16}$ Garber, D.P. and Willshire Jr., W.L., "En route noise levels from propfan test assessment airplane," NASA TP-3451, September 1994.

${ }^{17}$ Sutherland, L.C., "Review of experimental data in support of a proposed new method for computing atmospheric absorption losses," DOT-TST-75-87, May 1975.

${ }^{18}$ Begault, D.R. 3-D sound for virtual reality and multimedia. Academic Press, Inc., Chestnut Hill, MA, 1994.

${ }^{19}$ Zölzer, U., ed. DAFX - Digital audio effects. John Wiley \& Sons, Ltd., West Sussex, England, 2002.

${ }^{20}$ Kahrs, M. and Brandenburg, K., eds. Application of digital signal processing to audio and acoustics. Kluwer Academic Publishers, Norwell, MA, 1998.

${ }^{21}$ Gillian, R.E., "Aircraft noise prediction program user's manual," NASA TM-84486, January 1983.

${ }^{22}$ Stone, J.R., "Interim prediction method for jet noise," NASA TM X-71618, 1974.

${ }^{23}$ Stone, J.R. and Montegani, F.J., "An improved prediction method for the noise generated in flight by circular jets," NASA TM 81470, 1980.

${ }^{24}$ Grosveld, F.W., Sullivan, B.M., and Rizzi, S.A., "Temporal characterization of aircraft noise sources," Proceedings of the 42nd AIAA Aerospace Sciences Meeting, AIAA-2004-1029, Reno, NV, 2004.

${ }^{25}$ Heidmann, M.F., "Interim prediction method for fan compressor source noise," NASA TM X-71763, 1979.

${ }^{26}$ Zwicker, E. and Fastl, H. Psychoacoustics: facts and models. Springer-Verlag, Berlin, 1990. 